\title{
Spectrum generating algebra for the pure spinor superstring
}

\author{
Renann Lipinski Jusinskas \\ ICTP South American Institute for Fundamental Research, \\ Instituto de Física Teórica, UNESP - Universidade Estadual Paulista, \\ Rua Dr. Bento T. Ferraz 271, 01140-070, São Paulo, SP, Brasil \\ E-mail: renannlj@ift.unesp.br
}

ABSTRACT: In this work, a supersymmetric DDF-like construction within the pure spinor formalism is presented. Starting with the light-cone massless vertices, the creation/annihilation algebra is derived in a simple manner, enabling a systematic construction of the physical vertex operators at any mass level in terms of $\mathrm{SO}(8)$ superfields, in both integrated and unintegrated forms.

KEYwORDS: Superstrings and Heterotic Strings, Superspaces

ArXiv EPRINT: 1406.1902 


\section{Contents}

1 Introduction $\quad 1$

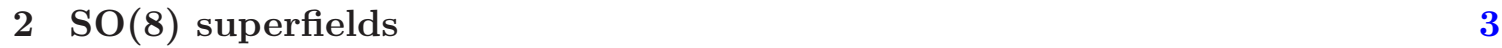

3 DDF operators: definition and algebra 5

$\begin{array}{lll}4 & \text { Physical spectrum } & 7\end{array}$

$\begin{array}{lll}5 & \text { Perspectives } & 10\end{array}$

\section{Introduction}

The pure spinor formalism debuted more than a decade ago [1] and remains the only one that allows Lorentz covariant computations with manifest supersymmetry. Its fundamental piece is a BRST-like charge

$$
Q_{\mathrm{BRST}}=\frac{1}{2 \pi i} \oint\left(\lambda^{\alpha} d_{\alpha}\right)
$$

that is nilpotent whenever $\lambda^{\alpha}$ is a pure spinor $\left(\lambda \gamma^{m} \lambda=0\right)$. Here,

$$
\begin{aligned}
d_{\alpha} & =p_{\alpha}+\frac{1}{2} \partial X^{m}\left(\gamma_{m} \theta\right)_{\alpha}+\frac{i}{8}\left(\theta \gamma^{m} \partial \theta\right)\left(\gamma_{m} \theta\right)_{\alpha}, \\
\Pi^{m} & =\partial X^{m}+\frac{i}{2}\left(\theta \gamma^{m} \partial \theta\right)
\end{aligned}
$$

are the invariants defined by the supersymmetry charge

$$
q_{\alpha}=\frac{1}{2 \pi} \oint\left\{-p_{\alpha}+\frac{1}{2} \partial X^{m}\left(\gamma_{m} \theta\right)_{\alpha}+\frac{i}{24}\left(\theta \gamma^{m} \partial \theta\right)\left(\gamma_{m} \theta\right)_{\alpha}\right\}
$$

and satisfy

$$
\begin{aligned}
\Pi^{m}(z) \Pi^{n}(y) & \sim-\frac{\eta^{m n}}{(z-y)^{2}}, \\
d_{\alpha}(z) \Pi^{m}(y) & \sim-\frac{\gamma_{\alpha \beta}^{m} \partial \theta^{\beta}}{(z-y)}, \\
d_{\alpha}(z) d_{\beta}(y) & \sim i \frac{\gamma_{\alpha \beta}^{m} \Pi_{m}}{(z-y)} .
\end{aligned}
$$

As usual, $m=0, \ldots, 9$ and $\alpha=1, \ldots, 16$ are the spacetime vector and chiral spinor indices, respectively. Observe that the fundamental length of the string is being fixed through $\alpha^{\prime}=2$ but it can be easily recovered by dimensional analysis. 
In spite of its unknown origin, the power of the formalism resides on the simple form of $Q_{\text {BRST }}$, which enables an elegant treatment of the cohomology in terms of superfields. For example, the massless open superstring states are in the cohomology of $Q_{\mathrm{BRST}}$ at ghost number one, represented by $U=\lambda^{\alpha} A_{\alpha}(X, \theta)$. Denoting the supersymmetric derivative as $D_{\alpha}=i \partial_{\alpha}-\frac{1}{2}\left(\gamma_{\alpha \beta}^{m} \theta^{\beta}\right) \partial_{m}$, the condition $\left\{Q_{\mathrm{BRST}}, U\right\}=0$ implies $D \gamma^{\text {mnpqr }} A=0$, which is the linearized equation of motion for the super Yang-Mills field $A_{\alpha}$. The gauge transformation $\delta A_{\alpha}=D_{\alpha} \Lambda$ is trivially reproduced in terms of BRST-exact states. The integrated form of the vertex $U$ is given by

$$
V=\frac{1}{2 \pi i} \oint\left\{\Pi^{m} A_{m}+i \partial \theta^{\alpha} A_{\alpha}+i d_{\alpha} W^{\alpha}+N^{m n} F_{m n}\right\},
$$

where the superfields $A_{m}, W^{\alpha}$ and $F_{m n}$ are defined as

$$
\begin{aligned}
A_{m} & \equiv \frac{1}{8 i}\left(D_{\alpha} \gamma_{m}^{\alpha \beta} A_{\beta}\right), \\
\left(\gamma_{m} W\right)_{\alpha} & \equiv\left(D_{\alpha} A_{m}+\partial_{m} A_{\alpha}\right), \\
F_{m n} & \equiv \frac{1}{2}\left(\partial_{m} A_{n}-\partial_{n} A_{m}\right) \\
& =\frac{i}{16}\left(\gamma_{m n}\right)_{\beta}^{\alpha} D_{\alpha} W^{\beta} .
\end{aligned}
$$

Both $U$ and $V$ were successfully used in loop amplitude computations and the results were shown to agree with the RNS superstring up to two loops [2]. On the other hand, the construction of the massive states is quite hard and only the first level of the open superstring has been studied in detail [3]. In fact, the proof that the pure spinor cohomology is equivalent to the light-cone Green-Schwarz spectrum was obtained in [4] through a complicated procedure, where the pure spinor variable was written in terms of $\mathrm{SO}(8)$ variables, involving an infinite chain of ghost-for-ghosts. Later, the equivalence of the pure spinor spectrum with the traditional superstring formalisms was demonstrated in different ways $[5,6]$, involving field redefinitions and similarity transformations, but an explicit superfield description of the massive states was still lacking.

Inspired on the work of Del Giudice, Di Vecchia and Fubini (DDF) for the bosonic string [7], this work presents a generalization of the spectrum generating algebra to the pure spinor superstring. A DDF construction within the pure spinor formalism was already discussed in [8]. However, the approach of Mukhopadhyay has two main differences. First, the gauge (Wess-Zumino) used there to build the DDF operators introduces unusual terms in the creation/annihilation algebra, demanding an extended argument for proving the validity of the construction, related to the orthonormality of the transverse Hilbert space. Second, the lack of an explicit expression for the DDF operators in [8], although sufficient for studying the D-Brane boundary states, makes the superfield description of the massive states incomplete. Here, a way to systematically obtain the pure spinor vertex operators at any mass level in terms of $\mathrm{SO}(8)$ superfields will be presented. Starting with the massless states in a particular light-cone gauge, which renders the massless vertices independent of half of the $\theta^{\alpha}$ components, the spectrum generating algebra will be shown to reproduce the expected superstring spectrum. As a by-product of this construction, Siegel's proposal 
for the massless superstring vertex [9] will be shown to give origin to a tachyonic state, reinforcing its quantum inequivalence with the massless vertex of the RNS string. In parallel, some particularities of the pure spinor approach will be discussed.

\section{$2 \mathrm{SO}(8)$ superfields}

It will be useful to establish the notation used here for the $\mathrm{SO}(8)$ decomposition. For any $\mathrm{SO}(1,9)$ vector $N^{m}$, the light-cone directions are represented by $\sqrt{2} N^{ \pm} \equiv\left(N^{0} \pm N^{9}\right)$ while the remaining spatial directions will be written as $N^{i}$, with $i=1, \ldots, 8$. The scalar product between $N^{m}$ and $P^{m}$ is simply $N^{m} P_{m}=-N^{+} P^{-}-N^{-} P^{+}+N^{i} P_{i}$. For a rank2 antisymmetric tensor $N^{m n}$, the $\mathrm{SO}(8)$ components are defined to be $N^{i j}, N^{i} \equiv N^{-i}$, $\bar{N}^{i} \equiv N^{+i}$ and $N \equiv N^{+-}$. The map between $\mathrm{SO}(1,9)$ and $\mathrm{SO}(8)$ spinor indices is given generically by

$$
\begin{array}{ccc}
\xi^{\alpha}=P_{a}^{\alpha} \xi^{a}+P_{\dot{a}}^{\alpha} \xi^{\dot{a}}, & \xi^{a}=P_{\alpha}^{a} \xi^{\alpha}, & \xi^{\dot{a}}=P_{\alpha}^{\dot{a}} \xi^{\alpha}, \\
\chi_{\alpha}=P_{\alpha}^{a} \chi_{a}+P_{\alpha}^{\dot{a}} \chi_{\dot{a}}, & \chi_{a}=P_{a}^{\alpha} \chi_{\alpha}, & \chi_{\dot{a}}=P_{\dot{a}}^{\alpha} \chi_{\alpha},
\end{array}
$$

where the $\mathrm{SO}(8)$ spinor indices are $a, b, \ldots$ and $\dot{a}, \dot{b}, \ldots$ (chiral and antichiral, respectively) running from 1 to 8 , and $\left\{P_{a}^{\alpha}, P_{\dot{a}}^{\alpha}, P_{\alpha}^{a}, P_{\alpha}^{\dot{a}}\right\}$ form a complete basis of projectors satisfying

$$
\begin{aligned}
P_{a}^{\alpha} P_{\alpha}^{b} & =\delta_{a}^{b}, & P_{\dot{a}}^{\alpha} P_{\alpha}^{\dot{b}} & =\delta_{\dot{a}}^{\dot{b}}, \\
P_{a}^{\alpha} P_{\alpha}^{\dot{a}} & =0, & \delta_{\beta}^{\alpha} & =P_{a}^{\alpha} P_{\beta}^{a}+P_{\dot{a}}^{\alpha} P_{\beta}^{\dot{a}} .
\end{aligned}
$$

In this language, the pure spinor constraint is translated to

$$
\lambda^{a} \lambda_{a}=\lambda^{\dot{a}} \lambda_{\dot{a}}=\lambda^{a} \lambda^{\dot{a}} \sigma_{a \dot{a}}^{i}=0
$$

where $\sigma_{a \dot{a}}^{i}$ are the $\mathrm{SO}(8)$ analogues of the Pauli matrices and satisfy

$$
\begin{aligned}
\sigma_{a \dot{a}}^{i} \sigma_{b \dot{a}}^{j}+\sigma_{b \dot{a}}^{i} \sigma_{a \dot{a}}^{j} & =2 \eta^{i j} \eta_{a b}, \\
\sigma_{a \dot{a}}^{i} \sigma_{a \dot{b}}^{j}+\sigma_{b \dot{a}}^{i} \sigma_{a \dot{a}}^{j} & =2 \eta^{i j} \eta_{\dot{a} \dot{b}}, \\
\sigma_{a \dot{a}}^{i} \sigma_{b \dot{b}}^{i}+\sigma_{b \dot{a}}^{i} \sigma_{a \dot{b}}^{i} & =2 \eta_{a b} \eta_{\dot{a} \dot{b}} .
\end{aligned}
$$

Here, $\eta_{i j}, \eta_{a b}$ and $\eta_{\dot{a} \dot{b}}$ are the $\mathrm{SO}(8)$ metrics of the vector and spinor indices. Since they are flat metrics with + signature, upper and lower $\mathrm{SO}(8)$ indices will not be distinguished in this work. Finally, the $\mathrm{SO}(1,9)$ gamma matrices will be written as

$$
\begin{aligned}
\left(\gamma^{i}\right)^{\alpha \beta} & \equiv P_{a}^{\alpha} \sigma_{a \dot{a}}^{i} P_{\dot{a}}^{\beta}+P_{a}^{\beta} \sigma_{a \dot{a}}^{i} P_{\dot{a}}^{\alpha}, & \left(\gamma^{i}\right)_{\alpha \beta} & \equiv P_{\alpha}^{a} \sigma_{a \dot{a}}^{i} P_{\beta}^{\dot{a}}+P_{\beta}^{a} \sigma_{a \dot{a}}^{i} P_{\alpha}^{\dot{a}}, \\
\left(\gamma^{-}\right)^{\alpha \beta} & \equiv \sqrt{2} P_{a}^{\alpha} P_{a}^{\beta}, & \left(\gamma^{-}\right)_{\alpha \beta} & \equiv-\sqrt{2} P_{\alpha}^{\dot{a}} P_{\beta}^{\dot{a}}, \\
\left(\gamma^{+}\right)^{\alpha \beta} & \equiv \sqrt{2} P_{\dot{a}}^{\alpha} P_{\dot{a}}^{\beta}, & \left(\gamma^{+}\right)_{\alpha \beta} & \equiv-\sqrt{2} P_{\alpha}^{a} P_{\beta}^{a},
\end{aligned}
$$

and can be shown to satisfy $\left\{\gamma^{m}, \gamma^{n}\right\}=2 \eta^{m n}$. Observe that the spinor projectors are being defined implicitily so their explicit form will never be required. 
In the construction to be presented, left and right-moving fields will be split. The only subtlety comes from the worldsheet scalars $X^{m}$, which will be written as

$$
X^{m}(z, \bar{z})=X_{L}^{m}(z)+X_{R}^{m}(\bar{z}) .
$$

This will be useful in studying holomorphic and anti-holomorphic sectors in an independent manner. The difference between open and closed strings will be discussed later, in the analysis of the spectrum.

Suppose one starts analyzing the unintegrated massless vertex with a definite momentum $P^{m}=\frac{1}{2 \pi} \oint \partial X^{m}$. From the Lorentz covariance of the theory, an equivalent state can be constructed with momentum $P^{+}=\sqrt{2} k$ and $P^{-}=0$ in another frame. The gauge

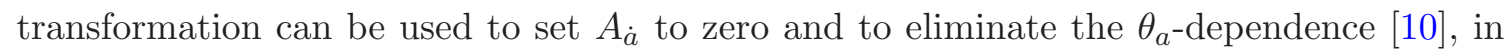
such a way that $\lambda^{\alpha} A_{\alpha}$ can be rewritten as $\lambda^{a} A_{a}=a^{i} U_{i}+\chi^{a} Y_{a}$, where $a^{i}$ and $\chi^{a}$ are the polarizations of the massless vector and spinor, respectively, and

$$
\begin{aligned}
U_{i}(z ; k) \equiv & e^{-i k \sqrt{2} X_{L}^{-}}\left\{\bar{\Lambda}_{i}+\left(\frac{k}{3 !}\right) \bar{\theta}_{i j} \bar{\Lambda}_{j}+\left(\frac{k^{2}}{5 !}\right) \bar{\theta}_{i j} \bar{\theta}_{j k} \bar{\Lambda}_{k}+\left(\frac{k^{3}}{7 !}\right) \bar{\theta}_{i j} \bar{\theta}_{j k} \bar{\theta}_{k l} \bar{\Lambda}_{l}\right\}, \\
Y_{a}(z ; k) \equiv & e^{-i k \sqrt{2} X_{L}^{-}}\left(\sigma^{i} \theta\right)_{a}\left\{\left(\frac{1}{2 !}\right) \bar{\Lambda}_{i}+\left(\frac{k}{4 !}\right) \bar{\theta}_{i j} \bar{\Lambda}_{j}+\left(\frac{k^{2}}{6 !}\right) \bar{\theta}_{i j} \bar{\theta}_{j k} \bar{\Lambda}_{k}+\left(\frac{k^{3}}{8 !}\right) \bar{\theta}_{i j} \bar{\theta}_{j k} \bar{\theta}_{k l} \bar{\Lambda}_{l}\right\} \\
& +e^{-i k \sqrt{2} X_{L}^{-}}\left(\frac{\lambda_{a}}{k}\right) .
\end{aligned}
$$

Here, $\bar{\Lambda}^{i} \equiv\left(\lambda^{a} \sigma_{a \dot{a}}^{i} \theta^{\dot{a}}\right), \bar{\theta}^{i j} \equiv\left(\theta^{\dot{a}} \sigma_{\dot{a} \dot{b}}^{i j} \dot{\theta}^{\dot{b}}\right)$ and $\sigma^{i j} \equiv \sigma^{[i} \sigma^{j]}$. To show the BRST-closedness of (2.4) note that

$$
\begin{aligned}
{\left[Q_{\mathrm{BRST}}, \bar{\theta}^{i j}\right] } & =2 i \bar{\Lambda}^{i j} \\
\left(\sigma_{i} \lambda\right)_{a} \bar{\theta}^{i j} & =-\left(\sigma_{i} \theta\right)_{a} \bar{\Lambda}^{i j}+3 \bar{\Lambda}\left(\sigma^{j} \theta\right)_{a} \\
\bar{\Lambda}_{i j} \bar{\Lambda}_{j} & =-3 \bar{\Lambda}_{i} \bar{\Lambda}
\end{aligned}
$$

with $\bar{\Lambda} \equiv\left(\lambda^{\dot{a}} \theta_{\dot{a}}\right)$ and $\bar{\Lambda}^{i j} \equiv\left(\lambda^{\dot{a}} \sigma_{\dot{a} \dot{b}}^{i j} \theta^{\dot{b}}\right)$. All of relevant properties follow from the $\mathrm{SO}(8)$ Fierz identities derived from (2.2), e.g.

$$
\sigma_{a \dot{a}}^{i} \sigma_{\dot{b} \dot{c}}^{i j}=-\sigma_{a \dot{b}}^{i} \sigma_{\dot{a} \dot{c}}^{i j}+2 \eta_{\dot{a} \dot{b}} \sigma_{a \dot{c}}^{j}-\eta_{\dot{b} \dot{c}} \sigma_{a \dot{a}}^{j}-\eta_{\dot{a} \dot{c}} \sigma_{a \dot{a}}^{j}
$$

In complete analogy, one is able to construct the massless light-cone vertices with momentum $P^{-}=\sqrt{2} k$ and $P^{+}=0$. The result is:

$$
\begin{aligned}
\bar{U}_{i}(z ; k) \equiv & e^{-i k \sqrt{2} X_{L}^{+}}\left\{\Lambda_{i}+\left(\frac{k}{3 !}\right) \theta_{i j} \Lambda_{j}+\left(\frac{k^{2}}{5 !}\right) \theta_{i j} \theta_{j k} \Lambda_{k}+\left(\frac{k^{3}}{7 !}\right) \theta_{i j} \theta_{j k} \theta_{k l} \Lambda_{l}\right\}, \\
\bar{Y}_{\dot{a}}(z ; k) \equiv & e^{-i k \sqrt{2} X_{L}^{+}}\left(\theta \sigma^{i}\right)_{\dot{a}}\left\{\left(\frac{1}{2 !}\right) \Lambda_{i}+\left(\frac{k}{4 !}\right) \theta_{i j} \Lambda_{j}+\left(\frac{k^{2}}{6 !}\right) \theta_{i j} \theta_{j k} \Lambda_{k}+\left(\frac{k^{3}}{8 !}\right) \theta_{i j} \theta_{j k} \theta_{k l} \Lambda_{l}\right\} \\
& +e^{-i k \sqrt{2} X_{L}^{+}}\left(\frac{\lambda_{\dot{a}}}{k}\right)
\end{aligned}
$$


where $\Lambda^{i} \equiv\left(\theta^{a} \sigma_{a \dot{a}}^{i} \lambda^{\dot{a}}\right)$ and $\theta^{i j} \equiv\left(\theta^{a} \sigma_{a b}^{i j} \theta^{b}\right)$. Introducing the polarizations of the massless vector, $a^{i}$, and spinor, $\xi^{\dot{a}}$, the unintegrated vertex operator in this case can be cast as $\lambda^{\dot{a}} A_{\dot{a}}=a^{i} \bar{U}_{i}+\xi^{\dot{a}} \bar{Y}_{\dot{a}}$, with

$$
\begin{aligned}
A_{\dot{a}}= & e^{-i k \sqrt{2} X_{L}^{+}}\left\{\delta_{i l}+\left(\frac{k}{3 !}\right) \theta_{i l}+\left(\frac{k^{2}}{5 !}\right) \theta_{i j} \theta_{j l}+\left(\frac{k^{3}}{7 !}\right) \theta_{i j} \theta_{j k} \theta_{k l}\right\} a^{i}\left(\sigma^{l} \theta\right)_{\dot{a}}+e^{-i k \sqrt{2} X_{L}^{+}}\left(\frac{\xi_{\dot{a}}}{k}\right) \\
& +e^{-i k \sqrt{2} X_{L}^{+}}\left\{\left(\frac{1}{2 !}\right) \delta_{i l}+\left(\frac{k}{4 !}\right) \theta_{i l}+\left(\frac{k^{2}}{6 !}\right) \theta_{i j} \theta_{j l}+\left(\frac{k^{3}}{8 !}\right) \theta_{i j} \theta_{j k} \theta_{k l}\right\}\left(\xi \sigma^{i} \theta\right)\left(\sigma^{l} \theta\right)_{\dot{a}} \cdot(2.10)
\end{aligned}
$$

The next step is to derive the expressions for the superfields of (1.7). Since the construction is very similar for $P^{+} \neq 0$ and $P^{-} \neq 0$, the latter will be used in order to illustrate the procedure. It may be useful to emphasize that $A_{a}=0$ and $D_{\dot{a}} A_{\dot{b}}=0$ (the dependence on $\theta_{\dot{a}}$ was removed) due to a gauge choice. Besides, $D_{a} A_{\dot{a}}=i A_{i} \sigma_{a \dot{a}}^{i}$. This can be seen from the Fierz decomposition of $D_{a} A_{\dot{a}}$, given by

$$
-i\left(D_{a} A_{\dot{a}}\right)=A_{i} \sigma_{a \dot{a}}^{i}+A_{i j k} \sigma_{a \dot{a}}^{i j k} .
$$

The last term is proportional to the linearized equation of motion for $A_{\alpha},\left(D \gamma^{+-i j k} A\right)=0$, so $A_{i j k}=0$. The first term, $A^{i}$, represents the non-vanishing vector components of the superfield given in (1.7a):

$$
\begin{aligned}
A_{i}= & e^{-i k \sqrt{2} X_{L}^{+}}\left\{\delta_{i j}+\left(\frac{k}{2 !}\right) \theta_{j i}+\left(\frac{k^{2}}{4 !}\right) \theta_{j k} \theta_{k i}+\left(\frac{k^{3}}{6 !}\right) \theta_{j l} \theta_{l k} \theta_{k i}+\left(\frac{k^{4}}{8 !}\right) \theta_{j m} \theta_{m k} \theta_{k l} \theta_{l i}\right\} a^{j} \\
& +e^{-i k \sqrt{2} X_{L}^{+}}\left\{\delta_{i j}+\left(\frac{k}{3 !}\right) \theta_{j i}+\left(\frac{k^{2}}{5 !}\right) \theta_{j k} \theta_{k i}+\left(\frac{k^{3}}{7 !}\right) \theta_{j l} \theta_{l k} \theta_{k i}\right\}\left(\xi \sigma^{j} \theta\right) .
\end{aligned}
$$

For $W^{\alpha}$, the $\mathrm{SO}(8)$ decomposition of $(1.7 \mathrm{~b})$ gives $W^{a}=0$ and $W^{\dot{a}}=-i k A^{\dot{a}}$. At last, the non-vanishing components of the super field strength are $\sqrt{2} F_{+i}=-\sqrt{2} F_{i+}=$ $-i k A_{i}$, completing all the blocks needed for the construction of the integrated massless vertex. Notice that the particular gauge of the approach presented here enables the simple component expansion of the $\mathrm{SO}(8)$ superfields of (2.10) and (2.12), which were already discussed in the work of Brink, Green and Schwarz [11].

\section{DDF operators: definition and algebra}

The gauge fixed version of (1.6) is given by

$$
\bar{V}_{\text {L.C. }}\left(k ; a^{i}, \xi^{\dot{a}}\right)=\frac{1}{2 \pi i} \oint\left\{\left(\Pi_{i}-i \sqrt{2} k \bar{N}_{i}\right) A^{i}+\left(i \partial \theta^{\dot{a}}+k d^{\dot{a}}\right) A_{\dot{a}}\right\},
$$

where

$$
\bar{N}^{i} \equiv N^{+i}=-\frac{1}{\sqrt{2}} \omega^{\dot{a}} \lambda^{a} \sigma_{a \dot{a}}^{i}
$$

As a consistency check, observe that

$$
\left[Q_{\mathrm{BRST}}, \bar{V}_{\mathrm{L} . \mathrm{C} .}\right]=-\frac{1}{2 \pi i} \oint\left\{\partial\left(\lambda^{\dot{a}} A_{\dot{a}}\right)+\sqrt{2} k^{2} \bar{N}^{i}\left(\lambda^{a} \sigma_{a \dot{a}}^{i} A^{\dot{a}}\right)\right\} .
$$


The first term inside the curly brackets is a total derivative whereas the last one vanishes due to the pure spinor constraint $\lambda^{a} \lambda_{a}=0$, cf. equation (2.1), as

$$
\begin{aligned}
\bar{N}^{i}\left(\lambda \sigma^{i}\right)_{\dot{a}} & =-\frac{1}{\sqrt{2}} \omega^{\dot{b}} \lambda^{a} \lambda^{b}\left(\sigma_{a \dot{a}}^{i} \sigma_{b \dot{b}}^{i}\right) \\
& =-\frac{1}{2 \sqrt{2}} \omega^{\dot{b}} \lambda^{a} \lambda^{b}\left(\sigma_{a \dot{a}}^{i} \sigma_{b \dot{b}}^{i}+\sigma_{a \dot{b}}^{i} \sigma_{b \dot{b}}^{i}\right) \\
& =-\frac{1}{\sqrt{2}} \omega_{\dot{a}}\left(\lambda^{a} \lambda_{a}\right) .
\end{aligned}
$$

Hence $\left[Q_{\mathrm{BRST}}, \bar{V}_{\mathrm{L} . \mathrm{C} .}\right]=0$. It might be helpful to point out that $\partial=\Pi^{+} \partial_{+}-i \partial \theta^{a} D_{a}$ whenever acting on superfields that depend only on $X^{+}$and $\theta^{a}$.

Defining the DDF operators $\bar{V}_{i}$ and $\bar{W}_{\dot{a}}$ through

$$
\bar{V}_{\text {L.C. }}\left(k ; a^{i}, \xi^{\dot{a}}\right) \equiv a^{i} \bar{V}_{i}(k)-i \xi^{\dot{a}} \bar{W}_{\dot{a}}(k),
$$

it will be demonstrated that

$$
\begin{aligned}
{\left[\bar{V}_{i}(k), \bar{V}_{j}(p)\right] } & =\sqrt{2} k \delta_{i j} \delta_{p+k} P^{+}, \\
{\left[\bar{V}_{i}(k), \bar{W}_{\dot{a}}(p)\right] } & =0, \\
\left\{\bar{W}_{\dot{a}}(k), \bar{W}_{\dot{b}}(p)\right\} & =\sqrt{2} \delta_{\dot{a} \dot{b}} \delta_{p+k} P^{+},
\end{aligned}
$$

which consists of a creation/annihilation algebra whenever acting on states with $P^{+} \neq 0$.

Although the following demonstration does not rely on the explicit computation of the (anti)commutators in (3.5), the explicit form of the DDF operators will be presented here for completeness:

$$
\begin{aligned}
\bar{V}_{i}(k)= & \frac{1}{2 \pi i} \oint\left\{\Pi_{i}-i \sqrt{2} k \bar{N}_{i}+\left(i \partial \theta^{\dot{a}}+k d^{\dot{a}}\right)\left(\sigma_{i} \theta\right)_{\dot{a}}\right\} \\
& +\frac{1}{2 \pi i} \oint\left\{\left(\frac{k}{2 !}\right) \theta_{i j}+\left(\frac{k^{2}}{4 !}\right) \theta_{i k} \theta_{k j}+\left(\frac{k^{3}}{6 !}\right) \theta_{i l} \theta_{l k} \theta_{k j}+\left(\frac{k^{4}}{8 !}\right) \theta_{i m} \theta_{m k} \theta_{k l} \theta_{l j}\right\} \Pi_{j} e^{-i k \sqrt{2} X_{L}^{+}} \\
& -\frac{k}{\sqrt{2} \pi} \oint\left\{\left(\frac{k}{2 !}\right) \theta_{i j}+\left(\frac{k^{2}}{4 !}\right) \theta_{i k} \theta_{k j}+\left(\frac{k^{3}}{6 !}\right) \theta_{i l} \theta_{l k} \theta_{k j}+\left(\frac{k^{4}}{8 !}\right) \theta_{i m} \theta_{m k} \theta_{k l} \theta_{l j}\right\} \bar{N}_{j} e^{-i k \sqrt{2} X_{L}^{+}} \\
& +\frac{1}{2 \pi i} \oint\left\{\left(\frac{k}{3 !}\right) \theta_{i l}+\left(\frac{k^{2}}{5 !}\right) \theta_{i j} \theta_{j l}+\left(\frac{k^{3}}{7 !}\right) \theta_{i j} \theta_{j k} \theta_{k l}\right\}\left(i \partial \theta^{\dot{a}}+k d^{\dot{a}}\right)\left(\sigma^{l} \theta\right)_{\dot{a}} e^{-i k \sqrt{2} X_{L}^{+}}, \\
\bar{W}_{\dot{a}}(k)= & \frac{1}{2 \pi} \oint\left\{-d^{\dot{a}}+\sqrt{2} \partial X^{+} \theta^{\dot{a}}+\Pi_{i}\left(\sigma^{i} \theta\right)_{\dot{a}}+\frac{i}{2}\left(\sigma^{i} \theta\right)_{\dot{a}}\left(\partial \theta^{\dot{c}} \sigma_{c \dot{c}}^{i} \theta^{c}\right)\right\} e^{-i k \sqrt{2} X_{L}^{+}} \\
& +\frac{1}{2 \pi} \oint\left\{\left(\frac{k}{3 !}\right) \theta_{j i}+\left(\frac{k^{2}}{5 !}\right) \theta_{j k} \theta_{k i}+\left(\frac{k^{3}}{7 !}\right) \theta_{j l} \theta_{l k} \theta_{k i}\right\}\left(\sigma^{j} \theta\right)_{\dot{a}} \Pi_{i} e^{-i k \sqrt{2} X_{L}^{+}} \\
& +\frac{k \sqrt{2}}{2 \pi i} \oint\left\{\delta_{i j}+\left(\frac{k}{3 !}\right) \theta_{j i}+\left(\frac{k^{2}}{5 !}\right) \theta_{j k} \theta_{k i}+\left(\frac{k^{3}}{7 !}\right) \theta_{j l} \theta_{l k} \theta_{k i}\right\}\left(\sigma^{j} \theta\right)_{\dot{a}} \bar{N}_{i} e^{-i k \sqrt{2} X_{L}^{+}} \\
& -\frac{1}{2 \pi i} \oint\left\{\left(\frac{k}{4 !}\right) \theta_{i l}+\left(\frac{k^{2}}{6 !}\right) \theta_{i j} \theta_{j l}+\left(\frac{k^{3}}{8 !}\right) \theta_{i j} \theta_{j k} \theta_{k l}\right\}\left(\sigma^{i} \theta\right)_{\dot{a}}\left(\partial \theta^{\dot{c}} \sigma_{c \dot{c}}^{l} \theta^{c}\right) e^{-i k \sqrt{2} X_{L}^{+}} \\
& +\frac{k}{2 \pi} \oint\left\{\left(\frac{1}{2 !}\right) \delta_{i l}+\left(\frac{k}{4 !}\right) \theta_{i l}+\left(\frac{k^{2}}{6 !}\right) \theta_{i j} \theta_{j l}+\left(\frac{k^{3}}{8 !}\right) \theta_{i j} \theta_{j k} \theta_{k l}\right\}\left(\sigma^{i} \theta\right)_{\dot{a}}\left(d^{\dot{c}} \sigma_{c \dot{c}}^{l} \theta^{c}\right) e^{-i k \sqrt{2} X_{L}^{+}} .
\end{aligned}
$$

Observe that $\bar{V}_{j}(k)^{\dagger}=\bar{V}_{j}(-k)$ and $\bar{W}_{\dot{a}}(k)^{\dagger}=\bar{W}_{\dot{a}}(-k)$, as expected. Besides $\bar{V}_{j}(0)=$ $-i P_{j}$ and $\bar{W}_{\dot{a}}(0)=q_{\dot{a}}$. 


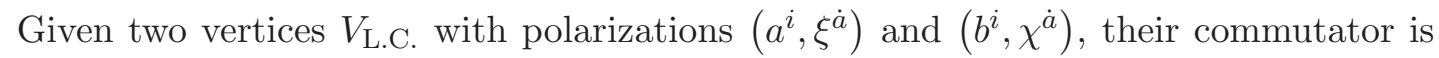
computed to be

$$
\begin{aligned}
{\left[\bar{V}_{\text {L.C. }}\left(k ; a^{i}, \xi^{\dot{a}}\right), \bar{V}_{\text {L.C. }}\left(p ; b^{i}, \chi^{\dot{a}}\right)\right]=} & -\frac{k}{2 \pi i} \oint\left\{A_{\dot{a}}(k, a, \xi) \partial A_{\dot{a}}(p, b, \chi)\right\} \\
& +\frac{1}{2 \pi i} \oint\left\{A_{i}(k, a, \xi) \partial A^{i}(p, b, \chi)\right\} \\
& -\frac{i k}{2 \pi i} \oint\left\{A_{\dot{a}}(k, a, \xi) \partial \theta^{a} D_{a} A_{\dot{a}}(p, b, \chi)\right\}
\end{aligned}
$$

and vanishes for $(k+p) \neq 0$, as the integrand is a total derivative:

$$
\begin{aligned}
A_{i}(k) \partial A_{i}(p)-k A_{\dot{a}}(k) \partial A_{\dot{a}}(p)-i k A_{\dot{a}} & (k) \partial \theta^{a}\left[D_{a} A_{\dot{a}}(p)\right]= \\
& =\frac{p}{k+p} \partial\left\{A_{i}(k) A_{i}(p)-k A_{\dot{a}}(k) A_{\dot{a}}(p)\right\} .
\end{aligned}
$$

Setting $(k+p)$ to zero (with $k \neq 0$ ), the expression inside the curly brackets in (3.8) is a constant written in terms of the polarizations, directly shown to be

$$
A_{i}(k, a, \xi) A_{i}(-k, b, \chi)-k A_{\dot{a}}(k, a, \xi) A_{\dot{a}}(-k, b, \chi)=a_{i} b_{i}+\left(\frac{1}{k}\right) \xi_{\dot{a}} \chi_{\dot{a}} .
$$

Note also that

$$
\begin{aligned}
A_{i}(k) \partial \theta^{a} D_{a} A_{i}(-k)= & -i k\left(\theta^{a} \partial \theta_{a}\right)\left(A_{i}(k) A_{i}(-k)-k A_{\dot{a}}(k) A_{\dot{a}}(-k)\right) \\
& -i \frac{k}{2}\left(\partial \theta_{i j}\right)\left(A^{i}(k) A^{j}(-k)+\frac{k}{4} A_{\dot{a}}(k) A_{\dot{b}}(-k) \sigma_{\dot{a} \dot{b}}^{i j}\right) .
\end{aligned}
$$

The second line of this equation is a total derivative and using this result together with (3.8) and (3.9), the right-hand side of (3.7) is rewritten in a very simple manner,

$$
\left[\bar{V}_{\text {L.C. }}\left(k ; a^{i}, \xi^{\dot{a}}\right), \bar{V}_{\text {L.C. }}\left(p ; b^{i}, \chi^{\dot{a}}\right)\right]=\delta_{p+k} \sqrt{2}\left(k a_{i} b_{i}+\xi_{\dot{a}} \chi_{\dot{a}}\right) P^{+},
$$

which implies the DDF algebra of (3.5) according to the definition (3.4).

In [8], using a different gauge for the massless vertices, the DDF-algebra was determined up to $\mathcal{O}\left(\theta^{2}\right)$. The DDF operators are BRST-closed by construction, so are their (anti)commutators. Since there is no other candidate to compose the massless pure spinor cohomology, $\mathcal{O}\left(\theta^{2}\right)$ must be BRST-exact and decouples naturally in the definition of the orthonormal basis presented by Mukhopadhyay. Another way to present this argument is recording that the difference between the DDF-operators of (3.4) and the ones presented in [8] is a simple BRST transformation (a gauge choice), implying that $\mathcal{O}\left(\theta^{2}\right)$ is BRST-trivial.

\section{Physical spectrum}

The closed string case will be discussed first. To understand the action of the operators $\bar{V}_{i}$ and $\bar{W}_{\dot{a}}$, consider the commutator $\left[\bar{V}_{i}(p), U_{j}(z ; k)\right]$. The OPE

$$
e^{-i p \sqrt{2} X_{L}^{+}}(y) e^{-i k \sqrt{2} X_{L}^{-}}(z) \sim(y-z)^{-2(k \cdot p)}: e^{-i \sqrt{2}\left(p X_{L}^{+}+k X_{L}^{-}\right)}:+\ldots
$$


will always appear and it is directly related to the mass levels of the superstring. Singlevaluedness of (4.1) will imply the discretization of $p$ in terms of $k: 2(k \cdot p) \in \mathbb{Z}$. This will be required in order for the operators to have any meaning at all, ${ }^{1}$ determining an acceptable operation for $\bar{V}_{i}$ and $\bar{W}_{\dot{a}}$ when commuting with $U_{j}$ and $Y_{a}$. Note also that whenever $k \cdot p \leq 0$, the commutator vanishes, as there are only simple poles coming from the contractions of $d_{\dot{a}}$ with the superfields $U_{j}$ and $Y_{a}$.

From the state-operator map, the ground states associated to the algebra (3.5) will be denoted by $|i ; k\rangle$ and $|a ; k\rangle$. They correspond, respectively, to the operators $U_{i}(z ; k)$ and $Y_{a}(z ; k)$ defined in (2.4). Then, the DDF-operators $\bar{V}_{i}\left(\frac{n}{2 k}\right)$ and $\bar{W}_{\dot{a}}\left(\frac{n}{2 k}\right)$, where $n \in \mathbb{Z}^{+}$, will generate the excited configurations. For example,

$$
\begin{array}{ll}
\bar{V}_{i}\left(\frac{1}{2 k}\right)|j ; k\rangle, & \bar{W}_{\dot{a}}\left(\frac{1}{2 k}\right)|a ; k\rangle, \\
\bar{V}_{i}\left(\frac{1}{2 k}\right)|a ; k\rangle, & \bar{W}_{\dot{a}}\left(\frac{1}{2 k}\right)|j ; k\rangle,
\end{array}
$$

are the first massive level of the holomorphic sector of the closed string. Clearly, the physical states are the direct (level-matched) product of the holomorphic and anti-holomorphic sectors, and the full vertex operators generated through this procedure will have momentum $P^{+}=-\sqrt{2} k$ and $P^{-}=-\frac{N}{\sqrt{2} k}\left(\right.$ with $\left.N \in \mathbb{Z}^{*}\right)$, satisfying the mass-shell condition

$$
m_{\text {closed }}^{2}=2 P^{+} P^{-}=2 N \text {. }
$$

Concerning supersymmetry, it is easy to demonstrate that

$$
\begin{aligned}
\left\{q_{a}, U_{i}(k)\right\} & =0, & {\left[q_{a}, \bar{V}_{i}(k)\right] } & =-i k \sigma_{a \dot{a}}^{i} \bar{W}_{\dot{a}}(k), \\
\left\{q_{\dot{a}}, U^{i}(k)\right\} & =k \sigma_{a \dot{a}}^{i} Y^{a}(k), & {\left[q_{\dot{a}}, \bar{V}_{i}(k)\right] } & =0, \\
{\left[q_{a}, Y_{b}(k)\right] } & =0, & \left\{q_{a}, \bar{W}_{\dot{a}}(k)\right\} & =i \sigma_{a \dot{a}}^{i} \bar{V}_{i}(k), \\
{\left[q_{\dot{a}}, Y_{a}(k)\right] } & =\sigma_{a \dot{a}}^{i} U_{i}(k), & \left\{q_{\dot{a}}, \bar{W}_{\dot{b}}(k)\right\} & =\sqrt{2} \eta_{\dot{a} \dot{b}} \delta_{k} P^{+},
\end{aligned}
$$

and the supersymmetric structure of the spectrum is trivially shown.

For the open string, the steps are almost the same. The difference arises in the definition of the integrated vertex operators, which will now be given in terms of an integral on the boundary of the disk. The analogue of the OPE (4.1) is

$$
e^{i p \sqrt{2} X_{L}^{+}}(z) e^{i k \sqrt{2} X_{L}^{-}}(y) \sim(z-y)_{*}^{-4(k \cdot p) *} e^{i \sqrt{2}\left(p X_{L}^{+}+k X_{L}^{-}\right) *}+\ldots,
$$

where the operators are now boundary normal ordered $\underset{* *}{* *}$. The single-valuedness condition will now be $8(k \cdot p) \in \mathbb{Z}$, as only half of the complex plane appears in the definition of the

\footnotetext{
${ }^{1}$ This is of course frame independent, but the light-cone frame used here is much easier to deal with, as unphysical polarizations (BRST-exact states) are straightforward to identify. The physical meaning of this quantization is deeply related to the Virasoro conditions, as it is clear in the bosonic string, for example. However, the gauge-fixing mechanism that provides the pure spinor formalism its BRST-like charge was just recently discovered [14], where a twistor like constraint replaces the usual Virasoro ones. That is why this quantization condition seems to be technical rather than fundamentally based.
} 
line integral. In other words, while the worldsheet coordinate $\sigma \in[0,2 \pi)$ for the closed string, in the open string $\sigma \in[0, \pi]$. Then, the mass levels of the open string will be

$$
m_{\text {open }}^{2}=2 P^{+} P^{-}=\frac{N}{2} .
$$

Therefore, the light-cone unintegrated vertex operators in the pure spinor superstring at any mass level can all be manufactured from chains of commutators between the DDF operators with $p=\frac{n}{2 k}(n>0)$ and the massless (ground) operators $U_{i}(z ; k)$ or $Y_{a}(z ; k)$. Being BRST-closed by construction, this shows that the cohomology includes the lightcone superstring spectrum. An interesting observation is that the generated states contain only half of the $\lambda^{\alpha}$ components, namely $\lambda^{a}$, as it is the one that appears in $U_{i}$ and $Y_{a}$, and the pure spinor contribution to $\bar{V}_{i}(p)$ and $\bar{W}_{\dot{a}}(p)$ comes from the Lorentz current $\bar{N}^{i}=-\frac{1}{\sqrt{2}} \lambda^{a} \omega^{\dot{a}} \sigma_{a \dot{a}}^{i}$. Clearly, if the ground states were chosen to be $\bar{U}_{i}(z ; k)$ and $\bar{Y}_{\dot{a}}(z ; k)$ in (2.9), the DDF operators would have been built out of the massless integrated vertices with $P^{+} \neq 0$ and the light-cone spectrum would only depend on $\lambda^{\dot{a}}$.

The versatility of the spectrum generating algebra also extends to the integrated vertex operators. In order to see that, the $P^{+} \neq 0$ version of the DDF-operators will be needed and denoted by $V_{i}(k)$ and $W_{a}(k)$. The procedure is completely analogous to the one presented above, just replacing $U_{i}(z ; k)$ and $Y_{a}(z ; k)$ by $V_{i}(k)$ and $W_{a}(k)$, respectively. Note that their explicit form can be easily recovered from (3.1), by defining

$$
\begin{aligned}
V_{\text {L.C. }}\left(k ; a^{i}, \xi^{a}\right) & =\frac{1}{2 \pi i} \oint\left\{\left(\Pi_{i}-i \sqrt{2} k N_{i}\right) A^{i}+\left(i \partial \theta^{a}+k d^{a}\right) A_{a}\right\} \\
& \equiv a^{i} V_{i}(k)-i \xi^{a} W_{a}(k)
\end{aligned}
$$

where $A_{i}$ and $A_{a}$ are the $\mathrm{SO}(8)$-covariant superfields in the frame $P^{-}=P^{i}=0$ and $P^{+}=\sqrt{2} k$ constructed out of (2.4). There is now a subtlety that comes from the operators $\bar{V}_{i}\left(\frac{-1}{2 k}\right)$ and $\bar{W}_{\dot{a}}\left(\frac{-1}{2 k}\right)$, since the simple pole argument used for the annihilation of the unintegrated vertices when $k \cdot p<0$ does not work anymore. In other words, the commutators

$$
\begin{array}{ll}
{\left[\bar{V}_{i}\left(\frac{-1}{2 k}\right), V_{j}(k)\right],} & {\left[\bar{W}_{\dot{a}}\left(\frac{-1}{2 k}\right), V_{j}(k)\right],} \\
{\left[\bar{V}_{i}\left(\frac{-1}{2 k}\right), W_{a}(k)\right],} & \left\{\bar{W}_{\dot{a}}\left(\frac{-1}{2 k}\right), W_{a}(k)\right\},
\end{array}
$$

are no longer guaranteed to vanish, which is potentially dangerous as they would imply physical vertices corresponding to states with $m^{2}<0$, that is, tachyonic states.

In the pure spinor formalism, the vanishing of the vertices (4.7) is related to the level of the ghost Lorentz algebra. Basically, the relevant OPE that will appear in the computation is

$$
\bar{N}^{i}(z) N^{j}(y) \sim-3 \frac{\eta^{i j}}{(z-y)^{2}}-\frac{N^{i j}-\eta^{i j} N}{(z-y)},
$$

and precisely the factor of -3 ensures the absence of tachyons in this DDF description. In other words, the level of the ghost Lorentz algebra implies that there is no simple pole in the OPE between the integrands of $V_{\text {L.C. }}$ and $\bar{V}_{\text {L.C. }}$ when $(4.7)$ is concerned. It is 
interesting to note that the pure spinor ghosts play no role at all in the derivation of the DDF algebra (3.5), which means that the light-cone version of the massless vertex proposed by Siegel obeys the same algebra and the vertices suggested in (4.7) would exist. Roughly speaking, the "incompleteness" of the vertex

$$
V_{\text {Siegel }}=\frac{1}{2 \pi i} \oint\left\{\Pi^{m} A_{m}+i \partial \theta^{\alpha} A_{\alpha}+i d_{\alpha} W^{\alpha}\right\},
$$

leads to the existence of a tachyon in the physical spectrum. Perhaps a clearer example is the DDF construction in the bosonic string. Defining

$$
\begin{aligned}
V_{\text {bos }}^{i}(k) & \equiv \frac{1}{2 \pi i} \oint \partial X_{i} e^{-i k \sqrt{2} X_{L}^{-}}, \\
\bar{V}_{\text {bos }}^{i}(k) & \equiv \frac{1}{2 \pi i} \oint \partial X_{i} e^{-i k \sqrt{2} X_{L}^{+}},
\end{aligned}
$$

it is easy to see the emergence of the tachyon vertex operator:

$$
\left[\bar{V}_{\text {bos }}^{i}\left(\frac{-1}{2 k}\right), V_{\text {bos }}^{j}(k)\right]=-\frac{\eta^{i j}}{2 \pi i} \underbrace{\oint \exp \left\{-i k \sqrt{2} X_{L}^{-}+\frac{i}{\sqrt{2} k} X_{L}^{+}\right\}}_{\propto V_{\text {tachyon }}} .
$$

The level of the Lorentz current can be argued to be an evidence of the quantum equivalence of the pure spinor vertex operator (1.6) with the RNS massless one [1]. The spectrum generating algebra presented here, more than supporting this equivalence, shows through a simple construction that the light-cone spectrum of the pure spinor superstring coincides with the ones of the RNS and the Green-Schwarz formalisms. It must be pointed out that this is not a demonstration that the DDF-states span the pure spinor cohomology. For further details, see [10].

\section{Perspectives}

In a deeper analogy with the RNS string [12], it might be interesting to investigate whether a full DDF algebra will also exist here. If this is the case, it is expected that Siegel's like constraints will appear as symmetry generators for the operators $\bar{V}_{i}$ and $\bar{W}_{\dot{a}}$ (resembling, for example, the action of $\bar{V}_{\text {bos }}^{-}$in the usual bosonic construction, that satisfy $\left.\left[\bar{V}_{\text {bos }}^{-}(p), \bar{V}_{\text {bos }}^{i}(k)\right] \propto \bar{V}_{\text {bos }}^{i}(p+k)\right)$. A better understanding of the cohomology structure of the pure spinor formalism may help clarify its equivalence with the RNS and GreenSchwarz superstrings, which is now far from evident.

The flat space construction of the spectrum generating algebra is also very appealing for it allows the determination of the whole physical spectrum by knowing just the massless vertex operators. It would be great if this same procedure could shed some light in the current knowledge about the superstring spectrum in curved backgrounds. Recently, the massless cohomology of the pure spinor superstring in $\mathrm{AdS}_{5} \times S^{5}$ was determined in the limit close to the AdS boundary [13], providing some ground for further studies on the generalization of the results presented here. 


\section{Acknowledgments}

This work was motivated by a parallel research project on a light-cone analysis of the pure spinor formalism [10]. I would like to thank Nathan Berkovits for very useful suggestions. I would like to thank also FAPESP grants 2009/17516-4 for financial support and 2011/11973-4 (ICTP-SAIFR) for providing a stimulating research environment.

Open Access. This article is distributed under the terms of the Creative Commons Attribution License (CC-BY 4.0), which permits any use, distribution and reproduction in any medium, provided the original author(s) and source are credited.

\section{References}

[1] N. Berkovits, Super Poincaré covariant quantization of the superstring, JHEP 04 (2000) 018 [hep-th/0001035] [INSPIRE].

[2] N. Berkovits and C.R. Mafra, Equivalence of two-loop superstring amplitudes in the pure spinor and RNS formalisms, Phys. Rev. Lett. 96 (2006) 011602 [hep-th/0509234] [INSPIRE].

[3] N. Berkovits and O. Chandía, Massive superstring vertex operator in D $=10$ superspace, JHEP 08 (2002) 040 [hep-th/0204121] [INSPIRE].

[4] N. Berkovits, Cohomology in the pure spinor formalism for the superstring, JHEP 09 (2000) 046 [hep-th/0006003] [INSPIRE].

[5] N. Berkovits, Relating the RNS and pure spinor formalisms for the superstring, JHEP 08 (2001) 026 [hep-th/0104247] [INSPIRE].

[6] N. Berkovits and D.Z. Marchioro, Relating the Green-Schwarz and pure spinor formalisms for the superstring, JHEP 01 (2005) 018 [hep-th/0412198] [INSPIRE].

[7] E. Del Giudice, P. Di Vecchia and S. Fubini, General properties of the dual resonance model, Annals Phys. 70 (1972) 378 [INSPIRE].

[8] P. Mukhopadhyay, DDF construction and D-brane boundary states in pure spinor formalism, JHEP 05 (2006) 055 [hep-th/0512161] [INSPIRE].

[9] W. Siegel, Classical superstring mechanics, Nucl. Phys. B 263 (1986) 93 [InSPIRE].

[10] N. Berkovits and R. Lipinski Jusinskas, Light-cone analysis of the pure spinor formalism for the superstring, JHEP 08 (2014) 102 [arXiv:1406.2290] [INSPIRE].

[11] L. Brink, M.B. Green and J.H. Schwarz, Ten-dimensional supersymmetric Yang-Mills theory with SO(8)-covariant light-cone superfields, Nucl. Phys. B 223 (1983) 125 [InSPIRE].

[12] R.C. Brower and K.A. Friedman, Spectrum generating algebra and no ghost theorem for the Neveu-Schwarz model, Phys. Rev. D 7 (1973) 535 [INSPIRE].

[13] N. Berkovits and T. Fleury, Harmonic superspace from the $\operatorname{AdS}_{5} \times S^{5}$ pure spinor formalism, JHEP 03 (2013) 022 [arXiv:1212.3296] [INSPIRE].

[14] N. Berkovits, Twistor origin of the superstring, arXiv:1409.2510 [INSPIRE]. 